\title{
Complete genomic sequence and phylogenomics analysis of Agrobacterium strain AB2/73: a new Rhizobium species with a unique mega-Ti plasmid
}

Marjolein J. G. Hooykaas and Paul J. J. Hooykaas*

\begin{abstract}
Background: The Agrobacterium strain AB2/73 has a unique host range for the induction of crown gall tumors, and contains an exceptionally large, over $500 \mathrm{kbp}$ mega Ti plasmid. We used whole genome sequencing to fully characterize and comparatively analyze the complex genome of strain $A B 2 / 73$, including its Ti plasmid and virulence factors.

Results: We obtained a high-quality, full genomic sequence of $A B 2 / 73$ by a combination of short-read Illumina sequencing and long-read Nanopore sequencing. The AB2/73 genome has a total size of 7,266,754 bp with 59.5\% GC for which 7012 genes (6948 protein coding sequences) are predicted. Phylogenetic and comparative genomics analysis revealed that strain AB2/73 does not belong to the genus Agrobacterium, but to a new species in the genus Rhizobium, which is most related to Rhizobium tropici. In addition to the chromosome, the genome consists of 6 plasmids of which the largest two, of more than $1 \mathrm{Mbp}$, have chromid-like properties. The mega Ti plasmid is $605 \mathrm{kbp}$ in size and contains two, one of which is incomplete, rep $A B C$ replication units and thus appears to be a cointegrate consisting of about $175 \mathrm{kbp}$ derived from an unknown Ti plasmid linked to $430 \mathrm{kbp}$ from another large plasmid. In pTiAB2/73 we identified a complete set of virulence genes and two T-DNAs. Besides the previously described T-DNA we found a larger, second T-DNA containing a $6 \mathrm{~b}$-like onc gene and the acs gene for agrocinopine synthase. Also we identified two clusters of genes responsible for opine catabolism, including an acc-operon for agrocinopine degradation, and genes putatively involved in ridéopine catabolism. The plasmid also harbours tzs, iaaM and iaaH genes for the biosynthesis of the plant growth regulators cytokinin and auxin.
\end{abstract}

Conclusions: The comparative genomics analysis of the high quality genome of strain AB2/73 provided insight into the unusual phylogeny and genetic composition of the limited host range Agrobacterium strain AB2/73. The description of its unique genomic composition and of all the virulence determinants in pTiAB2/73 will be an invaluable tool for further studies into the special host range properties of this bacterium.

Keywords: Agrobacterium, Crown gall, Ti plasmid, AB2/73, Limited host range, Opine, Phylogenomics, Wholegenome sequencing, Nanopore sequencing

*Correspondence: p.j.j.hooykaas@biology.leidenuniv.nl Institute of Biology, Leiden University, Leiden, The Netherlands

\section{Background}

Bacteria of the genus Agrobacterium are the causal agents of the neoplastic plant diseases crown gall and hairy root [1]. The genus is part of the Rhizobiaceae family and species were initially defined based on their phytopathogenic properties: A. tumefaciens for crown gall tumor-inducing 
bacteria, A. rhizogenes for hairy root-inducing bacteria and $A$. radiobacter for avirulent strains. However, this classification appeared to be based on plasmid-determined properties, with $\mathrm{Ti}$ plasmids responsible for the induction of crown gall and Ri plasmids responsible for induction of hairy root. Regardless of the presence of any of these plasmids, Agrobacterium strains could be divided into three biovars based on phenotypic and metabolic characteristics [2]. Today, new taxonomic species and genera have been defined based on genomic relationships of these bacteria, which largely correspond to the previously defined biovars. For biovar 1 bacteria, the genus name Agrobacterium is kept, although more than 13 different genomovars (species) are now distinguished within this genus, each with specific ecological adaptations [3]. Bacteria of the genus Agrobacterium typically have both a circular and a linear chromosome [4]. To biovar 2 bacteria the species name Rhizobium rhizogenes was given and to biovar 3 bacteria the name Allorhizobium vitis [5]. These very different bacteria may nevertheless contain virtually identical Ti plasmids. More recently, four new tumorigenic strains isolated from blackberry galls were found to belong to the genus Rhizobium, but differ from $R$. rhizogenes and were given the species name Rhizobium tumorigenes [6].

Ti plasmids contain one or more T-DNA regions, sections that are transferred to plant cells during infection [1]. The T-DNA genes are expressed in the transformed plant cells and convert them into tumor cells, which eventually form a crown gall tumor on the infected plant. Responsible T-DNA genes often include genes involved in the biosynthesis of auxin (iaaM, iaaH) and cytokinin $(i p t)$, as well as genes such as $6 \mathrm{~b}$ belonging to the phenotypic plasticity (plast) family that modulate plant growth in an unknown manner [7]. In crown galls, specific metabolites called opines are formed that can be specifically broken down by the infecting bacteria [8]. The formation of the opines is catalyzed by opine synthases encoded by the T-DNA. The opine catabolic genes are also encoded on the Ti plasmid, but in a region outside of the T-DNA. The Ti plasmids are often classified by the specific opine(s) formed in the tumor, e.g. octopine, nopaline, succinamopine Ti plasmids.

The T-DNA is transferred in a single stranded form (T-strand) into plant cells by a transfer system encoded by the virulence region on the Ti plasmid and which is evolutionary related to the bacterial inc $P$ plasmid broad host range conjugation system $[9,10]$. The virulence genes involved are distributed over several operons including operon virB1-11 encoding the Type IV secretion system (T4SS), required for T-DNA delivery. The virD1-2 and virC1-2 genes are necessary for DNA processing and $\mathrm{T}$-strand formation, and virD4 encodes the coupling protein that can bring T-strands to the T4SS. The genes in the virulence region are controlled by a 2-component system consisting of the histidine receptor kinase VirA and the transcriptional activator VirG, which is activated by small plant derived compounds such as acetosyringone [11]. During infection the bacterium delivers not only T-strands to plant cells, but also a series of effector proteins (VirE2, VirE3, VirD5, VirF) that facilitate transformation [12].

Ti plasmids are conjugative plasmids that can spread to other bacteria through a series of conjugative transfer genes $(t r a, t r b)$ that are activated in the presence of a specific opine [13]. Thus conjugative transfer can be observed in tumors, where these opines are abundantly formed [14]. Like most plasmids in Rhizobiaceae bacteria, Ti plasmids have a repABC unit for replication [15, 16]. In addition, they have still other, uncharacterized genes for unknown metabolic and other properties [10].

Most Ti plasmids confer on their hosts the ability to form tumors on a wide variety of dicot plants. However, some $A$. vitis strains can form tumors only on the vine and a limited number of other plant species. These limited host range (LHR) strains have a Ti plasmid with rearrangements in the T-DNA. An Agrobacterium strain with an extremely limited host range, called AB2/73, was isolated from a plant called Lippia canescens [17]. This bacterium can induce tumors on Lippia, and on squash and pumpkin, on which, remarkably, the normal wide host range (WHR) strains do not induce tumors [18], and thus AB2/73 may be a preferred gene vector for these plants. The Ti plasmid of strain AB2/73 was identified after conjugation in planta to a Ti plasmid-cured avirulent Agrobacterium strain, which rendered this strain tumorigenic. Tumors were induced by this transconjugant only on the same few plant species as by $A B 2 / 73$ itself, showing that the limited host range properties were determined by pTiAB2/73 [19]. Although most Ti plasmids are about $200 \mathrm{kbp}$ in size, the Ti plasmid from strain AB2/73 was reported to be over $500 \mathrm{kbp}$ in size [19]. Otten and Schmidt [20] used a vir probe and the T-DNA border repeat as a probe on a Southern blot with pTiAB2/73 plasmid DNA to identify the vir-region and the T-DNA of pTiAB2/73. The vir-region was partially sequenced, while the T-DNA, located in a $3.5 \mathrm{kbp}$ segment, was completely sequenced. It was found to contain two genes: gene $l s n$ encoding a protein related to nopaline synthase and a gene $l s o$ encoding a rolB-like Plast (phenotypic plasticity) protein involved in tumor formation [7]. The same authors could not find other T-regions with the same approach. However, it seems likely that an extra T-region with an agrocinopine synthase gene must be present in strain $\mathrm{AB} 2 / 73$, as agrocinopines have been detected in tumors induced by $A B 2 / 73$ [18]. 
Such an unknown T-region may very well contain other onc genes, as strain AB2/73 is more oncogenic and has a slightly wider host range for tumor induction than a strain that only introduces the lso gene into transformed plant cells [20].

We have now completely sequenced the genome of strain $A B 2 / 73$ and this unexpectedly revealed that strain AB2/73 neither belongs to the genus Agrobacterium, nor to any of the species $R$. rhizogenes, $R$. tumorigenes or $A$. vitis, but to a new, so far undescribed Rhizobium species. We found that the mega Ti plasmid of this strain has a size of $605 \mathrm{kbp}$. It has two rep $A B C$ replication units and appears to be a cointegrate of an (incomplete) unknown Ti plasmid with an entirely different, large plasmid. In the Ti plasmid portion of about $175 \mathrm{kbp}$, we found a complete set of vir genes and two regions surrounded by border repeats (T-DNAs) including a novel T-DNA with a 6b-like onc-gene and an acs gene for agrocinopine synthase. In the remainder of the Ti plasmid, we found a complete virulence region, a region containing genes for the biosynthesis of the plant growth regulators auxin (iaaM, iaaH) and cytokinin (tzs/ipt) and two regions containing genes for opine catabolism. Genes (tra, trb) for class I quorum-regulated conjugative $\mathrm{Ti}$ transfer, which are invariably present in Ti plasmids, were absent from pTiAB2/73, but other conjugative genes were present in the "non-Ti" part of the plasmid. We have used the pTiAB2/73 sequence for a detailed comparative analysis with other Ti and Ri plasmids.

\section{Results}

\section{General features of the genome of Agrobacterium} (Rhizobium) strain AB2/73

We obtained the full genomic sequence of $A B 2 / 73$ by Unicycler hybrid assembly of short-reads from Illumina sequencing and long-reads from MinION Nanopore sequencing. The $\mathrm{AB} 2 / 73$ genome has a total size of 7,266,754bp with $59.5 \%$ GC content for which 7012 genes (6948 protein coding sequences) are predicted. The chromosome has a size of 4,005,874 bp and contains the only three rDNA operons and all the 51 tRNA genes present in this bacterium. In addition to the chromosome, six DNA circles were identified with sizes of $1,332,287 \mathrm{bp}$, $1,068,678 \mathrm{bp}, \quad 605,540 \mathrm{bp}, \quad 158,599 \mathrm{bp}, \quad 56,335 \mathrm{bp}$ and $39,441 \mathrm{bp}$. These represent the (mega) plasmids previously seen on gels and called pAtAB2/73f-pAtAB2/73a [19]. The chromosome is the only genetic element encoding a DnaA replication initiation protein; the other DNA circles all have a rep $A B C$ system for replication as is most common for plasmids in the Rhizobiaceae family [15, 16]. For chromosome and plasmids, COG functional categories were assigned to predicted coding sequences, as can be seen on the circular maps in Figs. 1 and 2 and summarized for each of the circles in the bar charts in Fig. S1. The circular maps also show the GC content, GC skew, as well as the location of transposable elements. The smallest four replicons have a higher density of insertion-sequence (IS) elements, especially pAtAB2/73d (the Ti plasmid), which contains 47 IS elements (78 IS elements per million basepairs). While the GC content of the chromosome was $60.0 \%$, this was only slightly lower at 59.3 and $59.7 \%$ for the two $>1 \mathrm{Mbp}$ long plasmids, whereas it was $56.6-58.0 \%$ for the smaller plasmids. Both of the megabase-sized plasmids, like the primary chromosome, but unlike the smaller replicons, clearly have one part with mainly positive GC skew, whereas the other half shows mainly negative GC skew (Figs. 1 and 2). Such bias in guanines/cytosines composition (GC skew) across both DNA strands is present in most bacterial chromosomes $[21,22]$. This asymmetry is thought to (partially) develop over evolutionary time because of the mechanistic difference between leading and lagging strand synthesis during genome replication, whereby selection pressure leads to an enrichment of guanines in the leading strand. The AB2/73 megabase-sized plasmids thus appear to have been under such selective pressure long enough for this GC skew to develop. The similarity in GC content and GC skew to the primary chromosome suggests that these two $>1 \mathrm{Mbp}$ long plasmids may be chromids, mega-plasmids developing into secondary chromosomes [23].

\section{Comparative genomics and phylogenetic relatedness}

The strain AB2/73 was originally classified as a biotype 2 Agrobacterium, nowadays Rhizobium rhizogenes [19]. However, AB2/73 does not belong to the species R. rhizogenes. Its genome shares only $83.3 \% \mathrm{ANI}$ and $25 \% \mathrm{dDDH}$ with the two fully sequenced $R$. rhizogenes strains, K84 [24] and LBA9402 [25]. Neither does AB2/73 belong to the recently described species Rhizobium tumorigenes [6] with which it shares only 79.3\% ANI and $20.9 \% \% \mathrm{dDDH}$. The genome shows more similarity to that of Rhizobium tropici strain CIAT899 (89.8\% ANI, dDDH 38.6\%), that of Rhizobium sp. strain 11515TR (93.2\% ANI, dDDH 50\%), and especially that of the partially sequenced Rhizobium sp. strain YK2 (99.5\% ANI, 96.7\% dDDH). Strain $\mathrm{AB} 2 / 73$ thus belongs together with strain YK2 to a new Rhizobium species. A species tree showing the relationship of AB2/73 to other Rhizobium and Agrobacterium species is shown in Fig. S2. Whereas $R$. rhizogenes and $R$. tropici have only two replicons of $>1 \mathrm{Mbp}$, the Rhizobium sp. 11515TR genome, like AB2/73, has three replicons of $>1 \mathrm{Mbp}$ : in addition to its primary chromosome of 4,003,789 bp, it also has two replicons of around 1.5 Mbp. Not only the chromosome, but also these last two 


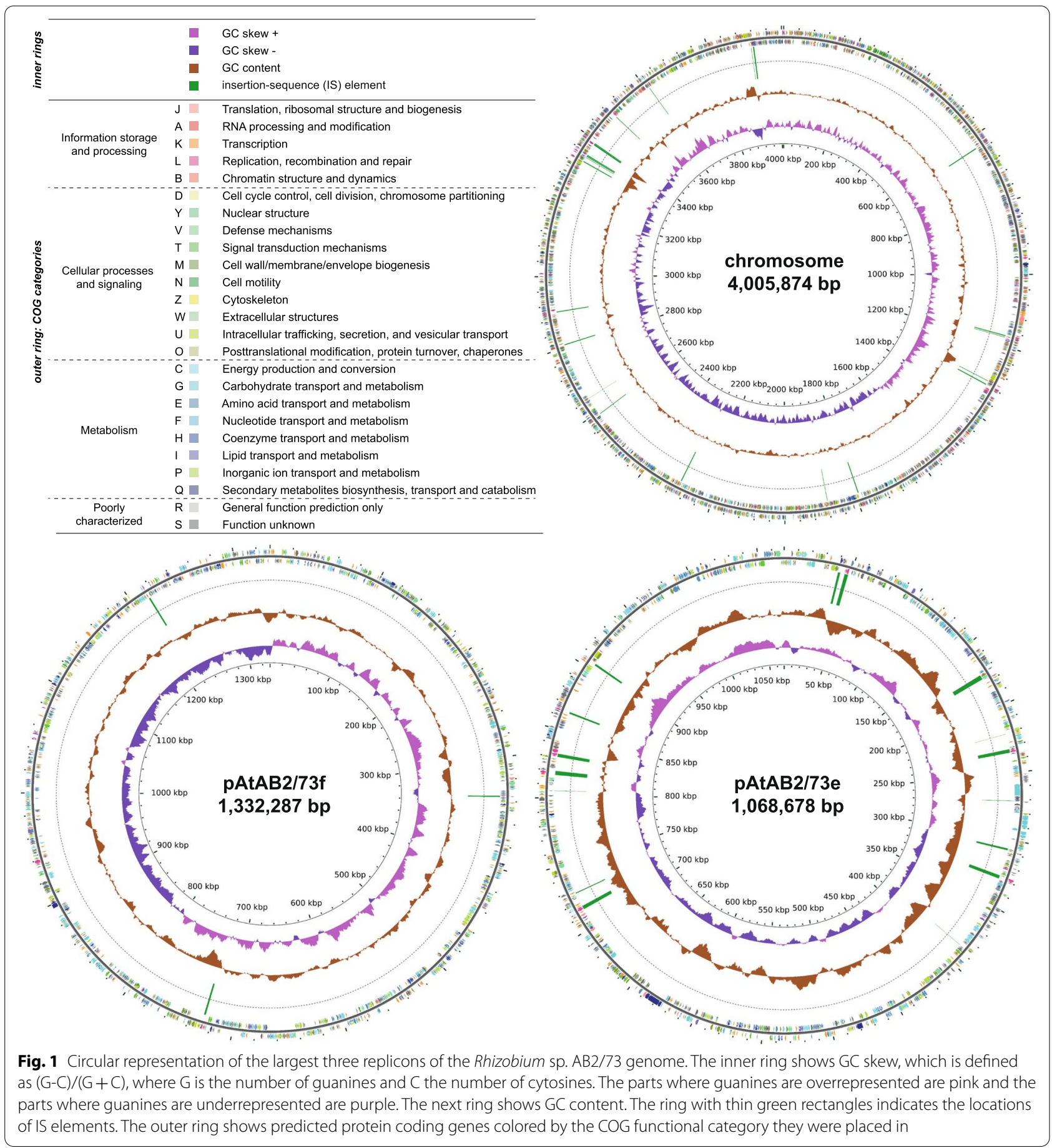

replicons show a significant overall similarity to replicons 2 and 3 of $A B 2 / 73$ (Fig. 3).

Over evolutionary time genes have been transferred from chromosome to chromids (and back) in the Rhizobiales and thus the location of genes in a bacterium can also provide evolutionary and phylogenetic insight [24]. To show the exchange of genes between the primary chromosomes and smaller replicons, gene presenceabsence heatmaps of the three largest AB2/73 replicons were constructed, which are shown in overview in Fig. 4, and with gene names added, for each of the three largest replicons separately in Figs. S3, S4 and S5. These figures show not only the presence-absence, but also by their color the replicon locations of homologs of the AB2/73 


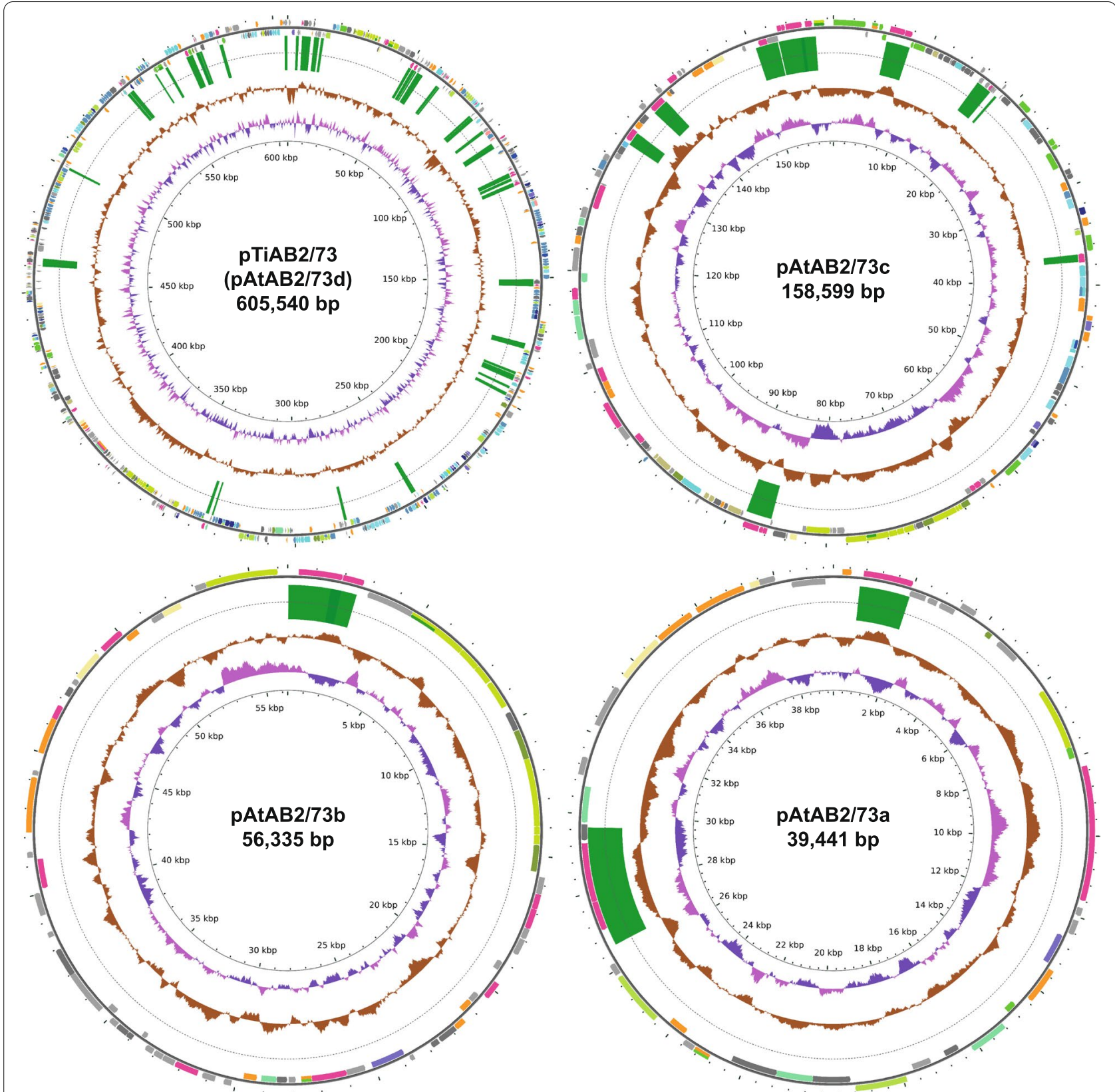

Fig. 2 Circular representation of the smallest four replicons of the Rhizobium sp. AB2/73 genome. For a description of the rings and COG functional categories see Fig. 1

genes in the genomes of other members of the Rhizobiaceae family with varying degrees of genome similarity to AB2/73. The species analyzed are listed in Table S1, while an overview of the numbers and sizes of replicons per genome is shown in Fig. S6. The heatmaps reveal patterns which are largely in agreement with the species tree, which is based on concatenated (chromosomal) protein alignments (shown above the heatmaps and in Fig. S2). That is, genomes which are part of the same clade appear to show (mostly) similar patterns of gene presence and/ or location.

The heatmap of the chromosomal genes (Fig. 4, Fig. S3) shows many (core) genes which are present in all genomes, but there are also clusters of genes which are only present in the Rhizobium species in the right half of the tree (including $R$. leguminosarum, $R$. phaseoli, $R$ etli, $R$. rhizogenes), but absent in the species in the left half of the tree (including Agrobacterium, Allorhizobium vitis and 


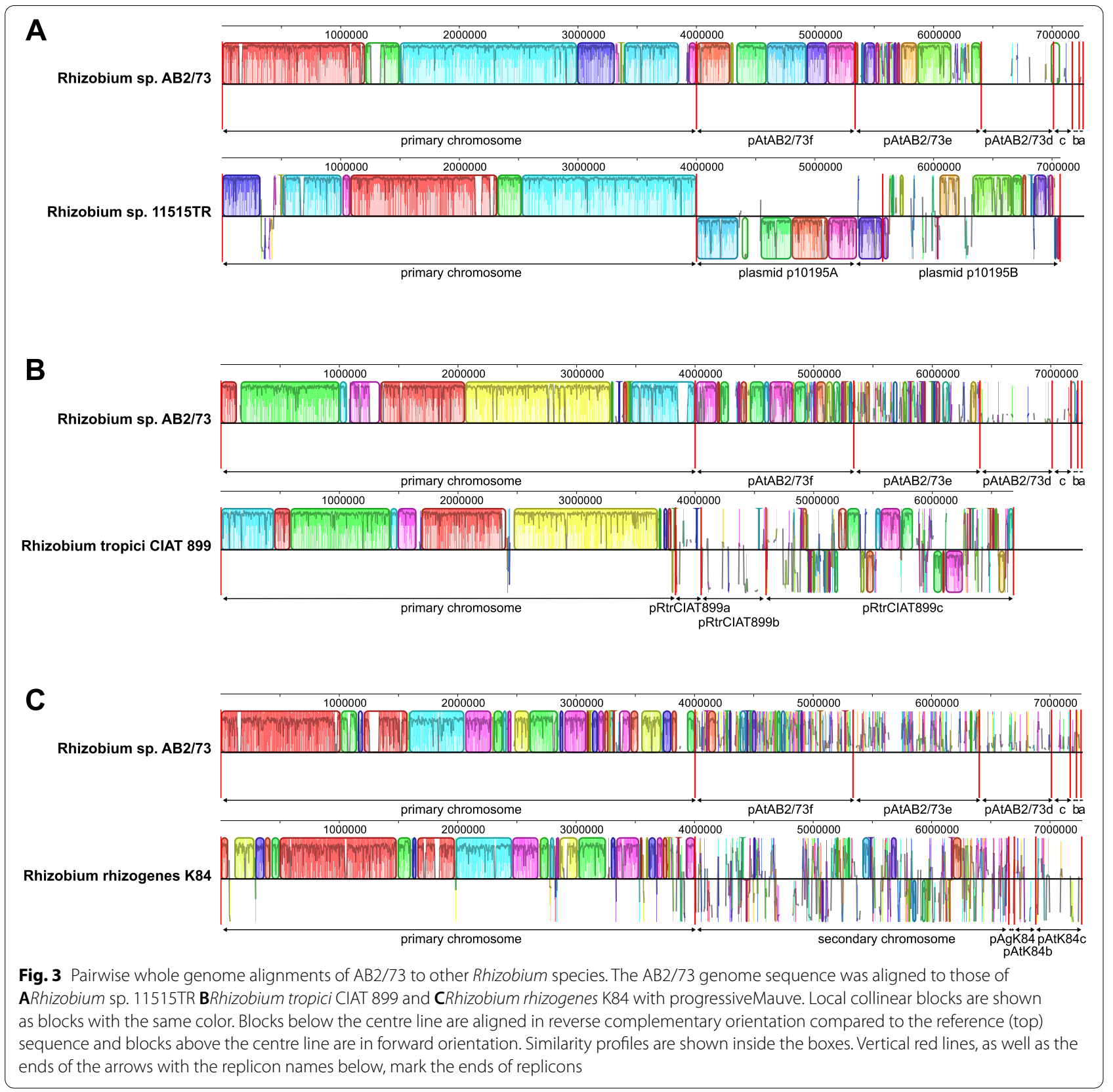

Neorhizobium). Finally, a number of chromosomal gene clusters are shared only with the species within the clade including $R$. tropici, $R$. rhizogenes, $R$. lusitanum and $R$. jaguaris (brown segment of the color bar) and some other gene clusters, including two representing putative integrated prophages, are unique for AB2/73. The heatmap also reveals the many chromosomal genes which in Agrobacterium (red segment of color bar) have moved to the linear chromosome in the course of evolution (replicon 2, blue). Some genes, such as the hut and panCB genes, are only located on the primary chromosome in the case of $A B 2 / 73$ and its closest relatives (brown segment of the color bar), but not in the other bacterial strains analyzed. This is probably as a result of a retro-transfer process, after initially having been present on the chromid of a Rhizobiales ancestor $[24,26]$. The clade including AB2/73, $R$. tropici, $R$. rhizogenes, $R$. lusitanum and $R$. jaguaris (brown segment of the color bar) shares with the Agrobacterium clade the location of the cyo genes (cytochrome-o-ubiquinol-oxidase-subunits) on the primary chromosome, in contrast to the other Rhizobium sp. in the right half of the tree which harbor these genes on plasmids. 
The second-largest replicon pAtAB2/73f, with a size of $1332 \mathrm{kbp}$, contains the cell division genes $\min C D E$ and the pca genes for protocatechuate degradation, which were transferred from chromosome to chromid before the radiation of the rhizobia [24]. pAtAB2/73f also carries genes for DNA recombination by end-joining (two genes for both $\mathrm{Ku}$ and LigD each), and for degradation of erythritol and thus growth on erythritol. There are fewer genes on pAtAB2/73f which are uniquely shared by the clade including $\mathrm{AB} 2 / 73, R$. tropici, and $R$. rhizogenes (brown segment of the color bar) as compared to chromosomal genes. However, the clade still shows a distinct color pattern in the heatmap. The neighboring clade containing Rhizobium grahamii BG7 and Rhizobium gallicum IE4872 (pink segment in the color bar) shows a similar color pattern in the pAtAB2/73f heatmap section and thus shares many of the pAtAB2/73f genes on their secondary replicons, which are very large in these bacteria. However, these genes are mostly either on the primary chromosome or on plasmids size-ranked third or fourth (still hundreds in kb in size) in the other Rhizobium clades (R. etli, R. phaseoli, and R. leguminosarum). Approximately half of the pAtAB2/73f genes are also present in Agrobacterium species, located either on the linear chromosome (also size-ranked second) or on their primary chromosome. On pAtAB2/73f there are a number of genes which are present in all five bacterial strains/ species in the AB2/73 - R. tropici subclade (left half of the brown segment in the color bar), but absent in its sister subclade ( $R$. rhizogenes, $R$. lusitanum and $R$. jaguaris), e.g. paaF - paaZ (a phenylacetate metabolic gene cluster).

In the pAtAB2/73e heatmap, the clade with $\mathrm{AB} 2 / 73, R$. tropici, and $R$. rhizogenes lacks a clear signature. However, most genes in pAtAB2/73e are shared with the third replicon of Rhizobium sp. 11515TR, in line with the whole genome alignment (Fig. 3), and also a significant portion is shared with replicons ranked third in Rhizobium sp. CCGE531 and CCGE532, which are also part of the AB2/73 - R. tropici subclade (left half of the brown segment). In contrast, however, the homologs of pAtAB2/73e genes are located on the relatively large, second-largest replicon in the $R$. tropici CIAT 899 genome. Perhaps a part of the third replicon has been joined with the second replicon in $R$. tropici. Homologs of the pAtAB2/73e genes are located both on primary chromosomes, large replicons/chromids and smaller plasmids or combinations of replicons in more distantly related Rhizobium genomes.

Overall, the data suggest that a common ancestor of AB2/73, Rhizobium sp. 11515TR, R. tropici and Rhizobium spp. CCGE531, CCGE532 harbored large pAtAB2/73f- and pAtAB2/73e-like replicons and that even though global collinearity has been lost (Fig. 3), the gene functions have, for a significant part, been maintained on the large replicons of its descendants. This conservation fits well with their other chromid-like features (GC content and GC skew similar to primary chromosome).

\section{Replicons pAtAB2/73a, pAtAB2/73b, and pAtAB2/73c} Plasmids pAtAB2/73b and pAtAB2/73c are probably conjugative because they contain the necessary conjugative transfer genes, while the smallest $39 \mathrm{kbp}$ plasmid pAtAB2/73a contains genes for mobilization proteins and for a coupling factor, but lacks the genes for a Type IV secretion system (T4SS). Thus the last plasmid may still be transmitted to new hosts using the T4SS encoded by one of the other plasmids. Plasmids with high similarity to pAtAB2/73a and pAtAB2/73c were not found in the genomes of any of the agrobacteria/rhizobia sequenced so far, but small portions of about $10 \%$ of these plasmids were found in several plasmids in bacteria of the Rhizobiaceae family. However, plasmids were found with high overall similarity to pAtAB2/73b. For example, the $A$. vitis plasmid pAtS4a, which is somewhat larger than pAtAB2/73b, shows $92 \%$ nucleotide identity in the shared segments (74\% coverage). Remarkably, in the $158 \mathrm{kbp}$ plasmid pAtAB2/73c there are genes for a

\footnotetext{
(See figure on next page.)

Fig. 4 Heatmap showing conservation of AB2/73 genes across Rhizobium/Agrobacterium genomes. The proteins of 125 Rhizobium and Agrobacterium species including AB2/73 were clustered with OrthoFinder as described in the Methods section. The presence of homologs of the AB2/73 proteins in other genomes is shown for the three largest replicons: the primary chromosome, pAtAB2/73f and pAtAB2/73e. Each row represents an orthogroup (gene) and each column a genome. The columns are ordered by the leaves of the concatenated protein-alignments-based species tree (Fig. S2). The heatmap is vertically split up into five sections based on the structure of the species tree shown at the top and the segments in the color bar indicate a further division of the tree into smaller subclades of related species/strains. The color bar colors are also used in the species tree to show these same clades. The heatmaps consist of small blocks of which the colors represent the relative size-rank of the replicon the gene is located on, as shown in the legend, where 1 represents the largest replicon. Thus, genes present only on the primary chromosome (always the largest replicon), are shown as a small black box, genes present on the semi-largest replicon (in $A$. tumefaciens and A. fabrum e.g. the linear chromosome) are shown in blue etc. The numbers of replicons per genome and their size is shown in Fig. S6. NB, multiple homologous genes of the same genome may be assigned to the same orthogroup. These genes are either categorized as replicon $x+$ other (s), or "multiple". For example, in the heatmaps primary chromosome section, genes present both on the primary chromosome and on another replicon are shown in grey, while genes present on multiple replicons but not on the primary chromosome are shown in brown
} 

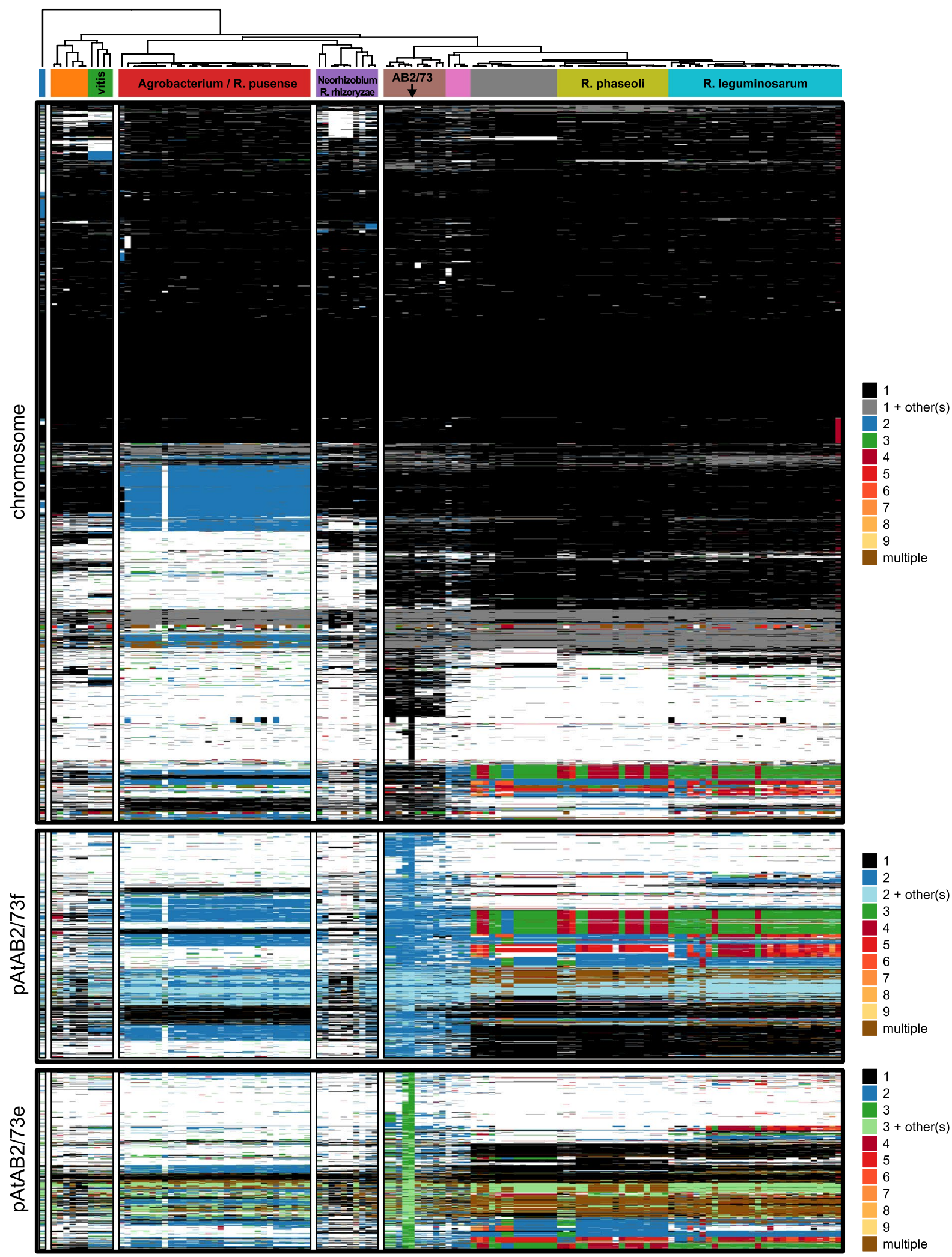

Fig. 4 (See legend on previous page.) 
restriction-modification system, and for DNA metabolism such as dnaQ (proofreading exonuclease), helD (helicase IV) and gyrA (topoisomerase IIA); in the $56 \mathrm{kbp}$ plasmid pAtAB2/73b genes encoding LigD and UmuCD (involved in SOS repair) are found.

\section{Plasmid pAtAB2/73d: the mega-Ti plasmid of AB2/73}

A plasmid estimated in size at the time to be about 500 kbp (pAtAB2/73d) was identified as the Ti plasmid, as this plasmid was seen in the recipient upon transfer of virulence [19]. We found that this plasmid corresponds to the 605,540 bp plasmid, which we identified by sequencing. A total of 575 protein coding sequences were found with an average size of $904 \mathrm{bp}$. No genes encoding transfer RNA (tRNA), or ribosomal RNA (rRNA) were found in pTiAB2/73. The plasmid backbone of pTiAB2/73 is very different from other Ti plasmids (Fig. 5). Compared to other $\mathrm{Ti}$ and Ri plasmids, it can be seen that Ti-like sequences together form an almost continuous segment of about $175 \mathrm{kbp}$. Beyond this Ti-like segment, a large $430 \mathrm{kbp}$ region is present with genes of largely unknown function with no counterpart in well-characterized Ti plasmids (Fig. 5). Within this $430 \mathrm{kbp}$ segment a rep $A B C$ operon is located with a $\operatorname{rep} C$ replicator gene which is only distantly related to that of other $\mathrm{Ti}$ plasmids so far characterized (Fig. S7). However, a second truncated repABC operon is present in the Ti-like segment with a rep $C$ gene that is related to that of other Ti plasmids (Fig. S7). Thus the pTiAB2/73 plasmid was probably formed by co-integration of two plasmids, an unknown Ti-plasmid and an entirely different plasmid. The Ti-like rep $A B C$ replicator was (partially) inactivated due to rearrangements that may have been caused by the insertion of a number of transposable elements. As a result, only a small portion of repA is still present in a fusion with a portion of $r e p B$, as can be seen when comparing this truncated rep $A B C$ operon to that of plasmid pAtCFBP4996a, to which the repA portion is most similar (Fig. S8). Ti and Ri plasmids described so far all possess a class I quorum-regulated conjugative transfer system with highly conserved conjugation genes located in whole or in part directly adjacent to the $\operatorname{rep} A B C$ genes [27]. However, in the Ti-like segment of pTiAB2/73, these highly conserved genes are not present next to the truncated rep $A B C$ unit, nor elsewhere in the plasmid. Such a quorum-driven conjugation system may have been lost during or after the cointegration event. Unrelated trb/virB genes for a Type IV secretion system (T4SS), a coupling protein and a protein with a putative relaxase/ mobilization nuclease domain are present in the "nonTi" portion of the plasmid. As shown for $\operatorname{trbB} / \mathrm{virB11}$ (Fig. S9) as an example, the T4SS genes found in the nonTi part of pTiAB2/73 do not cluster with the $t r b$ genes of
Ti plasmids, nor with the Ti virB genes, but similar genes can be found in a large number of Rhizobium (Sym) plasmids (Fig. S9). Apart from the T4SS genes, the 430 kbp large non-Ti portion of pTiAB2/73 contains mainly metabolic genes (Fig. 2) that have no counterpart in Ti plasmids or the Rhizobium (Sym) plasmids mentioned above (Fig. 5). It became apparent that pTiAB2/73 and in particular the Ti part, contains a large number of transposable elements (TEs, Fig. 2). One consequence of their action was truncation of the pTi-like rep $A B C$ operon, but they may also have been responsible for gross rearrangements including the loss of pTi-like tra/trb conjugation genes.

We found four sequences which differed at most 1 nucleotide from the left and right T-DNA border consensus sequences (Table S2) in pTiAB2/73. One set surrounded the previously identified T-region (T-DNA1) of $3.5 \mathrm{kbp}$ with the genes $l s n$ encoding an opine synthase and $l s o$, a rolB-like plast gene contributing to tumor formation [20]. It is unknown which opine is formed by the enzyme encoded by $l s n$. However, by protein sequence alignments we found that $A$. vitis plasmid pTiS4 harbors a gene highly similar (90.8\% identical at protein level) to $l s n$ in a fourth T-DNA (initially only three T-DNAs were reported in pTiS4 [28]). This T-DNA is very similar to T-DNA1 of pTiAB2/73 since both solely harbor a nos/lsnlike gene and a (homologous) rolB-like plast oncogene (Fig. 6; Fig. S10). It is known that in tumors induced by strain S4 the opines vitopine and ridéopine are produced [29]. While T-DNA2 of pTiS4 lacks opine synthase genes, T-DNA1 and T-DNA3 of pTiS4 contain vitopine synthase genes [28]. By inference it can therefore be concluded that the $l s n$ opine synthase gene in pTiAB2/73T-DNA1 and pTiS4 T-DNA4 must be responsible for the biosynthesis of the opine ridéopine. The finding that the opine synthase encoded by $l s n$ bears some similarity to nopaline synthase, is consistent with this hypothesis as nopaline and ridéopine are formed by a similar chemical reaction, the conjugation of $\alpha$-ketoglutarate with arginine and putrescine, respectively.

The pTiAB2/73 plasmid shares a large region of approximately $40 \mathrm{kbp}$, exclusively with the A. vitis pTiS4 and pTi1771 plasmids (Fig. 5, Fig. S10). This area, which includes both putative transporter and dehydrogenase genes, may be involved in opine uptake and catabolism. The $40 \mathrm{kbp}$ region shared by pTiS4, pTiAB2/73 and pTi1771 is located adjacent to T-DNA4 in pTiS4. It is tempting to speculate that this region may be involved in the degradation of ridéopine. Genes for a lactamase/ hydantoinase are also present, consistent with the fact that ridéopine can spontaneously form a lactam derivative [29]. A second region with the signature of an opine catabolic gene cluster in pTiS4, which is located close to 


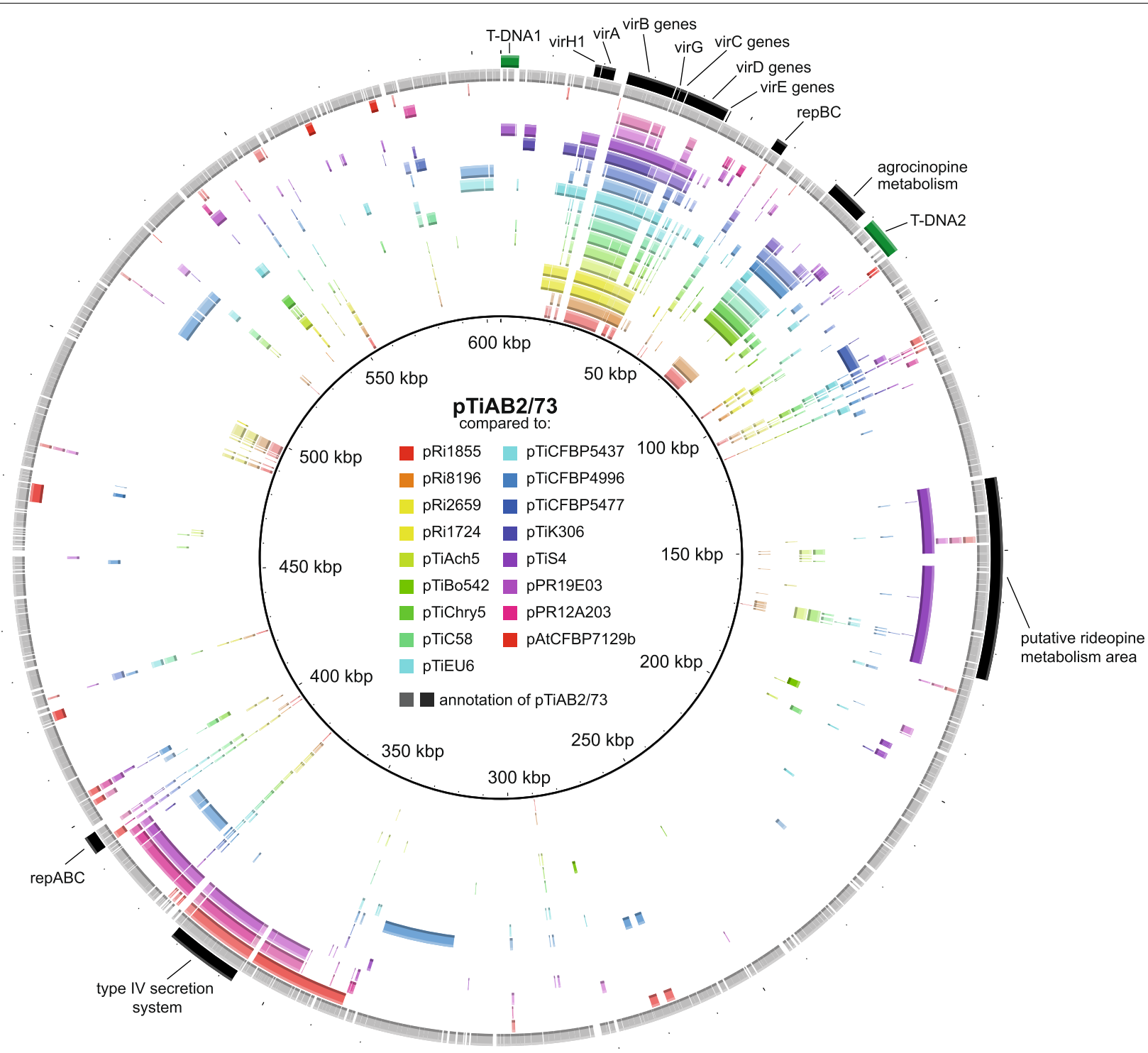

Fig. 5 Sequence comparison between pTiAB2/73 and various Ti, Ri, Sym and At plasmids. BLASTn hits are shown in concentric rings, from inner to outer ring: agropine Ri plasmid pRi1855 (accession CP044124), mannopine Ri plasmid pRi8196 (accession NZ_JAAMDI010000041.1), cucumopine Ri plasmid pRi2659 (accession NZ_CP019703), mikimopine Ri plasmid pRi1724 (accession NC_002575), octopine Ti plasmid pTiAch5 (accession NZ_CP007228), agropine Ti plasmid pTiBo542 (accession NC_010929), chrysopine Ti plasmid pTiChry5 (accession KX388536), nopaline Ti plasmid pTiC58 (accession NC_003065), succinamopine Ti plasmid pTiEU6 (accession KX388535), Ti plasmid pTiCFBP5473 (accession NZ_CP039694, from Agrobacterium larrymoorei), Ti plasmid pTiCFBP4996 (accession NZ_CM016551, mega Ti plasmid), Ti plasmid pTiCFBP5477 (accession NZ_CM016547, from A. larrymoorei), Ti plasmid pTiK306 (accession NZ_JABFNP010000003, from A. vitis), vitopine/ridéopine Ti plasmid pTiS4 (NC_01 1982, from A. vitis), Sym plasmid pPR19E03 (accession NZ_CP054030, from Rhizobium hidalgonense strain JKLM 19E), Sym plasmid pPR12A203 (accession NZ_CP054024, from Rhizobium indicum strain JKLM 12A2), plasmid PAtCFBP7129b (accession NZ_CP039925). Color intensity indicates the degree of sequence similarity. The second-last ring shows predicted coding sequences and in the outermost ring a number of areas and genes are indicated

T-DNA3 of pTiS4, has no counterpart in pTiAB2/73 or pTi1771 and is therefore likely involved in the degradation of vitopine.

The other T-region (T-DNA2) in pTiAB2/73 is $7.4 \mathrm{kbp}$ in size and contains the gene for agrocinopine synthase (acs) and a gene related to the $6 \mathrm{~b}$ oncogene. The gene content of the pTiAB2/73T-DNAs is shown schematically in Fig. 6. The composition of T-DNA2 is unique to $\mathrm{AB} 2 / 73$ and differs from all the T-DNAs so far identified [30]. The acs gene most closely resembles the acs genes present in agropine-type pTiBo542 and chrysopinetype pTiChry5, while the $6 \mathrm{~b}$ gene is most similar to that 


Fig. 6 Overview of genes in the T-DNA regions of pTiAB2/73 and pTiS4. The smallest T-DNA of pTiAB2/73 is also present in pTiS4, while the
Other T-DNA is not conserved. IS elements including predicted terminal repeats are shown as yellow blocks. The other rectangles are predicted
protein-coding genes, where blocks in the same color show similarity. Black triangles indicate the locations of the T-DNA border sequences. The
part right of the right border, with the iaaH, iaaM and ipt genes, is thus located outside of the second T-DNA but is shown to indicate the proximity
of these genes (which are usually located within the T-DNAs of other Ti plasmids, such as pTiS4)

present in nopaline Ti plasmids such as pTiKerr108 [31]. The presence of an acs gene in pTiAB2/73 was not unexpected as agrocinopines had previously been detected in tumors induced by this strain [18].

The presence of the acs gene for agrocinopine synthase in one of the T-DNAs is accompanied by an agrocinopine catabolic operon with genes $a c c R$ and an $a c c A-a c c G$ operon in the region between the Ti-like $\operatorname{rep} C$ and T-DNA2 (Fig. 5). The presence of these genes explains why strains containing pTiAB2/73 are sensitive to agrocin84 as previously established [19]. The catabolic acc genes of pTiAB2/73 are very similar to those of all other $\mathrm{Ri}$ and $\mathrm{Ti}$ plasmids conferring agrocinopine catabolism. However, the $a c c R$ regulator is more similar to that of the Ti plasmids, than to that of the Ri plasmids.

Surprisingly, just downstream of the T-DNA2 right border iaaM and iaaH genes for bacterial auxin biosynthesis and an ipt/tzs gene for bacterial cytokinin biosynthesis are located (Fig. 6). These genes were previously not picked up when the plasmid was probed with T-DNA $i a a H$, iaaM and ipt genes [19]. This can be explained by our finding that these genes are only distantly related to the T-DNA genes (Figs. S11, S12 and S13). Their presence in pTiAB2/73 may contribute to the bacterial virulence of strain $A B 2 / 73$, as in phytopathogenic bacteria such as Pseudomonas savastanoi [32].

The virulence operon was found to be located between the right border of T-DNA1 and the transposable elements that inactivated the $\operatorname{rep} A B C$ operon. At the right border end it starts with a virH1 gene followed by virA, virB1-11, virG, virC1-2, virD1-5 and ending with the virE1-3 genes. The virulence genes are most related to those present in A. vitis Ti plasmids (e.g. pTiS4 and pTiK306), in cucumopine (pRi2659) and mikimopine (pRi1724) Ri plasmids and in A. larrymoorei pTiCFBP5473 (Fig. S14; Table S3). While most of the pTiAB2/73 vir-genes showed high sequence identity with the vir-genes from these plasmids, the virE1-3 genes were much less conserved (Table S3). It was noteworthy that the virA gene of pTiAB2/73 lacks a vir-box and thus is not inducible by plant phenolics like that of $A$. vitis $\mathrm{Ti}$ plasmids and Ri plasmids. Lack of such vir-box in front of virA contributes to a host range restriction [33].

\section{Discussion}

Comparative bioinformatics analysis of the genome of Agrobacterium strain AB2/73 revealed that this strain does not belong to any of the classic tumor-inducing species, the Agrobacterium tumefaciens species group, Rhizobium rhizogenes or Allorhizobium vitis. Neither does strain AB2/73 belong to Neorhizobium sp. [34] or Rhizobium tumorigenes [6], new species with crown gall tumor-inducing bacteria. Strain AB2/73 rather belongs to a new bacterial species together with some so far unnamed and largely uncharacterized Rhizobium strains. The most closely related, well-characterized species is $R$. tropici with which it shares about $90 \%$ average nucleotide identity. In the Rhizobiales genes have moved from the primary chromosome to megaplasmids and back over evolutionary time [24]. Also genes have been lost or acquired from the plasmid pool, eventually leading to adaptation to particular niches. Gene presence/absence heat maps of the chromosomes and largest plasmids (chromids) give an impression of the genome dynamics and underlying gene mobility and also give an indication about genomic relationships in addition to the more classic nucleotide identity scores. In this way a subclade of rhizobia became apparent, containing $R$. rhizogenes, but also $R$. tropici and AB2/73 that is distinct from other rhizobia such as $R$. leguminosarum and $R$. etli. Strain $\mathrm{AB} 2 / 73$ differs in genomic makeup from $R$. rhizogenes and $R$. tropici in having two chromids instead of one.

That several species of the Rhizobiaceae family can become tumorigenic after receipt of a $\mathrm{Ti}$ plasmid is already known for a long time. Introduction of a Ti plasmid into Rhizobium trifolii, $R$. leguminosarum and $R$. phaseoli $[35,36]$ and also into the even more distantly related Phyllobacterium myrsinacearum [37] rendered these bacteria tumorigenic. However, tumors formed by these bacteria were often smaller than those induced by 
the Agrobacterium parental strain, probably because of a lack of co-evolution of these species with the Ti plasmid, and in some cases a recipient Rhizobium such as Sinorhizobium meliloti remained avirulent after receipt of a Ti plasmid [38]. Nevertheless, S. meliloti, Sinorhizobium fredii, Mesorhizobium loti and Ensifer adhaerens were later developed as gene vectors for plants by introducing a disarmed Ti plasmid and a binary vector into these bacteria $[39,40]$.

Sequencing of Rhizobium etli strain CFN42 revealed interestingly that one of its plasmids, $\mathrm{p} 42 \mathrm{a}$, naturally contains a full set of Ti-like virulence genes, [41] and subsequent introduction of a binary vector converted this strain in a gene vector for plants [42]. Plasmid p42a was reported to lack a T-DNA, but nevertheless may be derived from a Ti plasmid. In agreement with this hypothesis we found five sequences which differed at most 1 nucleotide from the left and right T-DNA border consensus sequences (Table S4). Between border repeat 4 and 5 genes are present including a gene annotated as an octopine/nopaline dehydrogenase, which may encode an opine synthase expressed in transformed plant cells. During the preparation of our manuscript the presence of putative border repeat 4 and an ocs-like gene in p42a was reported by Otten [30]. Therefore, it seems that a Ti plasmid transferred into this $R$. etli strain may have been inactivated over time by deletion of much of its T-DNAs suggesting that this bacterium may not be adapted well enough to compete with agrobacteria in the tumor niche. Also one should therefore be cautious in using this strain as a gene vector, as the segments of the p42a plasmid surrounded by border repeats may also end up in the transgenic plants besides the binary vector T-DNA selected.

Our finding of two sets (one intact, one truncated) of rep $A B C$ units suggests that pTiAB2/73 was formed by co-integration of two plasmids, a Ti plasmid and a much larger other plasmid. The Ti plasmid part contains relatively many transposable elements, which probably have caused rearrangements including deletion of some parts of the original Ti plasmid. For example, the Ti repABC operon is truncated and the Ti conjugation genes are all missing. Plasmid pTiAB2/73 is possibly still conjugative by a set of conjugation genes present in the non-Ti part. When AB2/73 was coinoculated in plants with a Ti plasmid cured recipient strain, a few transconjugants were obtained that had acquired the Ti plasmid [19]. When analyzed for plasmid content they had acquired not only pTiAB2/73, but also either pAtAB2/73b or pAtAB2/73c. Thus transfer may have been mediated by the putative conjugative system of pAB2/73 itself or by one of these plasmids, which we found to contain a full set of conjugative genes. As observed in other bacteria, transfer can then occur after cointegration of these plasmids in the donor, followed by transfer and resolution of the cointegrate in the recipient. Cointegration can be mediated by a transposase or an integrase present in one of the plasmids, or by the presence of homologous sequences shared by pTiAB2/73 and the conjugative plasmids [ 43 , 44].

Previously only one T-DNA could be discovered in the pTiAB2/73 by using the border repeat as a probe on a Southern blot with the Ti plasmid DNA [20], but we have identified a second T-DNA (T-DNA2) by using the border repeat as a query on the whole genome sequence. This revealed in total four border repeats in pTiAB2/73, surrounding the two T-DNAs. The presence of a second T-DNA with a $6 \mathrm{~b}$ onc gene may now explain why AB2/73 itself is more virulent on plants than a bacterium carrying the individual T-DNA1 with the single lso onc gene. The presence of T-DNA2 in pTiAB2/73 also explains the previously reported presence of agrocinopines in tumors induced by $\mathrm{AB} 2 / 73$ [18] as T-DNA2 contains the gene for agrocinopine synthase. T-DNA2 is unique to AB2/73, but T-DNA1 can also be found in the $A$. vitis pTiS4 plasmid ([30], our results), where it is present as the fourth T-DNA in addition to the three T-DNAs previously described [28].

While by Southern analysis it appeared that pTiAB2/73 lacked some detectable homology with the auxin ( $i a a H$, iaaM) and cytokinin biosynthetic (ipt) T-DNA genes [19], remarkably, such genes were still present, but not in either of the two T-DNAs, but in the region next to T-DNA2. Not only was an ipt / tzs-like gene present there, but $i a a H$ and $i a a M$ genes were also found, which code for enzymes that together can form the auxin indole acetic acid (IAA). Such iaa-genes are common in other phytopathogenic bacteria such as Pseudomonas savastanoi, where the release of IAA from the bacteria can cause gall formation [32]. The iaa-genes in pTiAB2/73 may originate directly from another bacterium or were previously present in a T-DNA, but have been displaced outside the T-DNA by DNA rearrangements. When we aligned the encoded proteins and constructed phylogenetic trees, we found that the AB2/73 iaaH and iaaM encoded proteins are much closer related to those of phytopathogenic bacteria such as Dickeya (more than $60 \%$ identity) than to those encoded by T-DNAs (around 35-50\% identity) (Figs. S11 and S12). It is likely therefore that the AB2/73 genes are not derived from a degraded T-DNA.

Strain AB2/73 was isolated from a crown gall on Lippia canesens and can induce tumors on very few other plants [17]. Interestingly, these plants include some squashes that cannot be transformed by standard agrobacteria [18]. The host range of strain AB2/73 is linked to the Ti plasmid; upon transfer to the Ti-cured laboratory strain C58, 
this strain became tumorigenic with the same limited host range as AB2/73 [19]. Previous research has been conducted on factors leading to a limited host range in A. vitis LHR Ti plasmids. The limited host range (LHR) in these strains could be extended by adding the ipt gene to the T-DNA of these strains, indicating that cytokinin production in the transformed plant cells is required for tumor proliferation in some host species [45, 46]. Thus, the absence of an ipt gene in the T-DNAs of pTiAB2/73 may be an important reason for its limited host range. However, Ti plasmid genes related to a limited host range have also been found in the virulence region [47]. The introduction of the virA gene from a WHR strain into an LHR A. vitis strain expanded the host range with Kalanchoe daigremontiana [47]. The requirement for a WHR Ti virA gene for tumor induction was due not so much to a different biological activity of the histidine kinase encoded by the WHR virA gene, but rather to a difference in expression due to the presence of a vir-box in the virA promoter [33]. The addition of such a vir-box to the promoter of the LHR Ti virA gene was sufficient to expand the host range for tumor induction with Kalanchoe daigremontiana [33]. The pTiAB2/73 plasmid has a complete virulence region with all essential vir-genes, but indeed, like $A$. vitis LHR strains, contains a virA gene without a vir-box. This can be seen in Table S5, which shows that a vir box in the virA promoter is present in almost all Ti and Ri plasmids, but is missing not only in the $A$. vitis Ti plasmids, but also in pTiAB2/73 and in the cucumopine and mikimopine Ri plasmids, the host range of which has not yet been described in detail. Why strain AB2/73 is tumorigenic on squashes in contrast to standard Agrobacterium strains could be related to the two plast oncogenes present in its T-DNAs. It was demonstrated that the $6 \mathrm{~b}$ gene of octopine strains by itself can mediate tumorigenicity on certain Kalanchoe species [48], while 6b genes from different strains can have a wide range of oncogenic properties $[7,49]$. It will be very interesting to test the oncogenic properties and host range of the $\mathrm{AB} 2 / 73$ onc genes.

\section{Conclusions}

Whole genome sequencing followed by a detailed comparison of the genome of 'Agrobacterium' strain AB2/73 with that of other bacteria of the Rhizobiaceae family revealed that $\mathrm{AB} 2 / 73$ belongs to a new Rhizobium species, which is most related to $R$. tropici and some other, still unnamed rhizobia. In the rhizobia, genes have often migrated from chromosome to plasmids (and sometimes back) over evolutionary time. Heat maps made to compare the position of the genes on the chromosome and the two chromids of AB2/73 with those of their homologs in other rhizobia revealed unique patterns, distinguishing AB2/73 along with R.tropici, $R$. jaguaris, R. lusitanum, and $R$. rhizogenes, which together also form a separate clade in the species tree, from other bacteria of the Rhizobiaceae family. The virulence of AB2/73 turned out to be due to the presence of a unique $605 \mathrm{kbp}$ mega-Ti plasmid, evolved from a cointegrate between a Ti plasmid and a much larger, unrelated plasmid. The description of its virulence genes, T-DNAs and opine catabolic genes will give direction to future studies regarding the unique host range of this bacterium.

\section{Methods \\ Organism}

Agrobacterium strain AB2/73 (LBA9200 in our collection) was obtained from prof. E. W. Nester (Seattle, USA). The bacterium was grown on TY medium (Difco tryptone $5 \mathrm{~g} / \mathrm{l}$, Difco yeast extract $3 \mathrm{~g} / \mathrm{l}, \mathrm{CaCl} 2.6 \mathrm{H} 2 \mathrm{O} 1.3 \mathrm{~g} / \mathrm{l}$ ) as described by Beringer [50]. The bacterium was tested for virulence by puncturing the plant stems of Nicotiana glauca with a sterile wooden toothpick that had been dipped into a colony of the bacterium.

\section{DNA sequencing}

Genomic DNA was isolated using QIAGEN Genomic-tip gravity-flow columns. The genomic sequence of $A B 2 / 73$ was determined using a combination of Illumina and Oxford Nanopore Technologies platforms. Nanopore sequencing was done in house, but Illumina sequencing was performed at the Leiden Genome Technology Center (LGTC) of the Leiden University Medical Center (Leiden, The Netherlands), where TruSeq DNA Libraries were sequenced on an Illumina HiSeq 2000 machine. The Oxford Nanopore sequencing library was generated with 200 ng DNA using the SQK-RBK004 Rapid Barcoding Kit. The library was pooled with another library, followed by in-house sequencing on a MinION flow cell (version R9.4.1). After basecalling with Albacore (version 2.3.4) the reads were demultiplexed (with Epi2me). The total yield for AB2/73 was 1,158,758 reads, totaling $5,160,617,317$ bp (with quality > Q7), with a N50 read length of $8263 \mathrm{bp}$ ( $710 \mathrm{x}$ coverage).

\section{Genome assembly and annotation}

Nanopore reads were end-trimmed and filtered on average quality $(>\mathrm{Q} 10)$ and length $(>10 \mathrm{~kb})$ with NanoFilt (200-fold coverage after filtering). A total of 6,021,213,100-nucleotide paired-end Illumina reads were quality and adapter trimmed using Cutadapt (70-fold coverage). Hybrid assembly was performed using Unicycler version 0.4.7. The eighth contig, representing the bacteriophage PhiX genome sequence, spiked-in during Illumina library preparation, was removed. The complete genome sequence of AB2/73 was deposited in GenBank under accession numbers CP067071-CP067077. The raw 
reads are deposited in the Sequence Read Archive under accessions numbers SRR13775335, SRR13775336. Microbial Genome Atlas (MiGA), TypeMat, and NCBI Prok were used to identify related genomes from the NCBI RefSeq and Prokaryotic Genomes databases. TypeMat also provided estimations of genome completeness and contamination. Based on the presence of essential genes, the TypeMat estimate of genome completeness was $99.1 \%$. ANI values were calculated with fastANI [51] and Digital DDH values with GGDC 2.1, whereby distances are inferred from identities/HSP (high scoring segment pairs) length for pairwise comparison of $\mathrm{AB} 2 / 73$ with other genomes [52]. The genome was annotated with NCBI Prokaryotic Genome Annotation Pipeline (PGAP [53]), as well as with eggnog-mapper v2 [54]. From the latter tool we obtained the assignments of proteins to Clusters of Orthologous Groups (COG) functional categories. ISEScan was used to annotate insertion sequences [55].

\section{Comparative genomics}

Full genome alignments were performed with progressiveMauve [56]. Schematics of T-DNA regions, rep(A)BC operons and vir regions were generated with the R package genoplotR [57]. BLASTn comparisons between plasmids TiAB2/73 and pTiS4 and other plasmids were performed and visualized with BLAST Ring Image Generator (BRIG) [58]. Orthogroups of homologous proteins were inferred with OrthoFinder version 2.3.12 (all-versus-all DIAMOND search followed by MCL clustering) [59]. For comparisons of $\mathrm{Ti}$ and $\mathrm{Ri}$ plasmid encoded proteins, protein sequences from $\mathrm{Ti}$ and $\mathrm{Ri}$ plasmids (extracted from Genbank files) were clustered. For the heatmaps, proteins from 125 Rhizobium and Agrobacterium genomes (listed in Table S1), and an in house sequenced Ochrobactrum strain (named strain LBA8980) were clustered. Pseudogenes were excluded. Genomes were selected based on NCBI taxonomic classification in the Rhizobium/Agrobacterium group and level of completeness (complete genomes only). The genomes were from organisms of genera Rhizobium, Neorhizobium, Agrobacterium and Allorhizobium (since Agrobacterium vitis is nowadays officially called Allorhizobium vitis). The species tree in Fig. 2 was inferred with FastTree 2.1.10 based on a (trimmed) concatenated protein alignment of 1165 single-copy genes generated by OrthoFinder. The OrthoFinder orthogroups table was processed with $\mathrm{R}$ to generate tables summarizing the location of genes on the genomes' replicons. Briefly, the protein identifiers were converted to (lists of) replicon ranks and this matrix was then merged to a table containing the AB2/73 locus tags (keeping only orthogroups present in the respective AB2/73 replicons). Single orthogroups are represented multiple times per matrix in case more than one AB2/73 gene has been assigned to it. For Fig. 4 the rows were clustered. A dissimilarity matrix was calculated based on Gower's distances, followed by Ward clustering. The R package ComplexHeatmap was used for visualizing the heatmaps.

Protein alignments were performed with MAFFT version 7.471, L-INS-I method [60] and visualized with pyBoxshade. Percentage identities of Vir proteins in Table S3 were calculated with the $\mathrm{R}$ package seqinr (based on the multiple sequence alignments). The VirB11/TrbB maximum likelihood tree was inferred with FastTree 2.1.10 (after MAFFT protein alignment). The RepC maximum likelihood tree was inferred with IQ-TREE 2.0.3 (after MAFFT alignment and TrimAI trimming, JTT + R7 model). Trees were visualized with the Python library ETE toolkit (version 3) [61]. Part of the work was performed using the ALICE computer resources provided by Leiden University.

\section{Abbreviations}

ANI: Average nucleotide identity; COG: Clusters of orthologous groups; dDDH: Digital DNA-DNA hybridization; HSP: High scoring segment pair; IS: Insertion sequence; LHR: Limited host range; TE: Transposable element; WHR: Wide host range.

\section{Supplementary Information}

The online version contains supplementary material available at https://doi. org/10.1186/s12866-021-02358-0.

Additional file 1: Figure S1. COG functional category classification of AB2/73 proteins. Shown is the number of proteins assigned to each COG functional category. Proteins falling into multiple categories were counted once for each category. On the right a description of the functional categories is given.

Additional file 2: Figure S2. Core genome phylogenetic tree. A phylogenetic tree of 125 Agrobacterium, Allorhizobium, Rhizobium and Neorhizobium species was inferred based on the concatenated protein alignments of 1165 single-copy genes. Ochrobactrum strain LBA8980 was used as an outgroup. The tree's branch colors are used in the color bars of the heatmaps (Fig. 4, Figs. S3, S4 and S5) and in the replicon sizes bar chart (Fig. S6) to indicate corresponding genomes. NB, not every clade contains equally closely related bacterial species. The $R$. leguminosarum/R. indicum clade consists of rather closely related species (short branch lengths), in contrast to the orange and purple clades which have rather long branch lengths, but were not further subdivided in order to keep a clear view. The red clade, besides all Agrobacterium species, contains a number of Rhizobium species. This is not unexpected, since of these, Rhizobium pusense and Rhizobium oryzihabitans harbor (like A. tumefaciens and A. fabrum) a linear chromosome and R. pusense has also been named Agrobacterium genomospecies G2. Rhizobium sp. NIBRBAC000502774 shows a strange pattern in the heatmaps (Fig. 3, Figs. S3, S4 and S5). Detailed investigation of this genome assembly was beyond the scope of this study, but in the genome taxonomy database (GTDB) it is listed as an Agrobacterium species, with a CheckM completeness score of only $84.09 \%$.

Additional file 3: Table S1. List of genomes used for analyses. Genomes are listed which were used for protein clustering, species tree construction and building the gene presence heatmaps.

Additional file 4: Figure S3. Heatmap showing conservation of AB2/73 chromosomal genes across Rhizobiaceae genomes. This figure is based 
on the same data as Fig. 4. However, in this larger version, rows were not clustered by similarity but ordered by the location of the genes in the AB2/73 chromosome.

Additional file 5: Figure S4. Heatmap showing conservation of pAtAB2/73f genes across Rhizobiaceae genomes. This figure is based on the same data as Fig. 4. However, in this larger version, rows were not clustered by similarity but ordered by the genes' location in pAtAB2/73f.

Additional file 6: Figure S5. Heatmap showing conservation of pAtAB2/73e genes across Rhizobiaceae genomes. This figure is based on the same data as Fig. 4. However, in this larger version, rows were not clustered by similarity but ordered by the genes' location in pAtAB2/73e.

Additional file 7: Figure S6. Stacked bar chart showing Agrobacterium/ Rhizobium genome and replicon sizes. The text color and order of bars are as per the leaves of the species tree in Fig. S2. The replicon rank colors correspond to those used in the heatmaps.

Additional file 8: Figure S7. RepC phylogenetic tree. A maximum likelihood phylogenetic tree was constructed from the RepC protein sequences from 125 Rhizobium and Agrobacterium genomes. The resulting tree was midpoint rooted. The scale bar shows the number of amino acid changes per site. The tree was annotated with protein descriptions written as organism|replicon name|locus tag. AB2/73 proteins are printed in bold. Ti plasmid proteins are shown in red text, Ri plasmid proteins in orange text and Sym plasmid sequences in green. The black leaf nodes represent plasmid encoded proteins, whereas chromosomal proteins have blue leaf nodes (using the genome assemblies annotation as 'chromosome' or 'plasmid'). Most (but not all) Ti plasmid RepC proteins are clustered together in a group indicated with red background. pTiAB2/73 harbors two repC genes. RepC encoded by the gene with locus tag I8E17_31105 (of the rep $B^{\prime}$-repC operon) lies in the group with the Ti plasmid RepC proteins, whereas the other RepC (of the repABC operon) groups with RepC proteins of some other non-Ti plasmids. RepC encoded by plasmids pAtAB2/73e and pAtAB2/73f cluster with RepC proteins from replicons of genomes to which $A B 2 / 73$ is most related according to the species tree.

Additional file 9: Figure $\mathbf{S 8}$. $\mathrm{pT}$ TAB2/73 repB of the repBC operon is truncated. A comparison is shown of pTiAB2/73 rep genes (locus tags I8E17_31100, 18E17_31105) with the repABC operon of plasmid pAtCFBP4996a. The red ribbons show tBLASTx hits $(e<0.001)$, with color intensity indicating the degree of sequence identity (darker $=$ higher similarity). The C-terminal part of I8E17_31100 ("repB") is conserved, yet the $\mathrm{N}$-terminus shows similarity to the $\mathrm{N}$-terminus and an internal part of the repA gene of pAtCFBP4996a. The gene I8E17_31100 thus appears to be a truncated repB fused to fragments of repA.

Additional file 10: Figure S9. VirB11/TrbB phylogenetic tree. A maximum likelihood phylogenetic tree was constructed based on VirB11/TrbB protein sequences from 125 Rhizobium and Agrobacterium genomes. The tree was midpoint rooted. The scale bar shows the number of amino acid changes per site. The tree was annotated with protein descriptions written as organism|replicon name|locus tag. Ti plasmid proteins are shown in red text, Ri plasmid proteins in orange text and Sym plasmid sequences in green. AB2/73 proteins are printed in bold. TrbB sequences form a separate clade (the part with the yellow background). Generally, $\mathrm{Ti}$ and Ri plasmids have a trb region, so the TrbB clade includes Ti and Ri plasmid sequences, however a pTiAB2/73 TrbB homolog is lacking. The VirB1 1 sequences from the vir regions of Ti and Ri plasmids also cluster together (clade with very light blue background), including a pTiAB2/73 protein (locus tag 18E17_30995). The rest of the proteins in the tree (darker blue background) are also named 'VirB11'but these are not derived from Ti plasmid virB operons. The only exception is the second pTiAB2/73 VirB11 homolog. The gene encoding this protein (locus tag l8E17_32535) is located in the non-Ti-like part of pTiAB2/73. This protein clusters with sequences mostly encoded by Rhizobium (indicum, leguminosarum, hidalgonense and etli) Sym plasmids. Some of these Sym plasmids, like pTiAB2/73, have a second VirB11 homolog which is rather similar to vir region-containing VirB11 (green names in very light blue background). The similarity of PTiAB2/73 vir region virB genes and the second set of virB-like genes to two such Sym plasmids, as well as an example of a plasmid which only shows similarity of the virB-like operon, can be seen in Fig. 5 (rings pPR19E03, pPR12A203 and pAtCFBP7129b, respectively).

Additional file 11: Table S2. T-DNA border sequences found in pTiAB2/73.

Additional file 12: Figure S10. Ridéopine metabolism region presumably conserved across pTiS4, pTiAB2/73 and pTi1771. The sequence of pTiS4 (accession NC_011982) is compared to various Ti and Ri plasmid sequences. BLASTn hits are shown in concentric rings, from inner to outer ring: nopaline Ti plasmid pTiC58 (accession NC_003065), octopine Ti plasmid pTiAch5 (accession NZ_CP007228), agropine/succinamopine Ti plasmid pTiBo542 (accession NC_010929), agropine Ri plasmid pRi1855 (accession CP044124), mikimopine Ri plasmid pRi1724 (accession NC_002575), Ti plasmid pTi1771 (unknown opine type, unpublished, $A$. vitis strain NCPPB 1771) and pTiAB2/73 (accession CP067074). The DNA region adjacent to pTiS4 T-DNA region 4, and the nopaline-like opine synthase gene within T-DNA4 (Isn), are conserved (only) in pTiAB2/73 and pTi1771. Since A. vitis 54 produces the opines vitopine and ridéopine, and since the T-DNA regions 1-3 harbour either vitopine synthase or no opine synthase, the Isn gene in T-DNA4 likely encodes a ridéopine synthase, and the adjacent region likely harbors the genes required for ridéopine import and catabolism.

Additional file 13: Figure $\mathbf{S 1 1}$. laaH/Tms2-like protein from pTiAB2/73 is more similar to non-T-DNA sequences than to T-DNA-encoded laaH. Alignment of Indoleacetamide hydrolase/amidase sequences from pTiAB2/73, from two Rhizobiaceae family members (Rhizobium tumorigenes and Allorhizobium vitis, $87 \%$ identical), two betaproteobacterial amidases (Dickeya dianthicola, Trinickia symbiotica, 63\% identical) and iaaH/tms2 from the T-DNAs of the Ti plasmids of A. tumefaciens strains C 58 , Ach5 (LBA4213) and S4 (32-34\% identical). Residues identical in the majority of sequences are shown with black shading, residues similar in the majority of sequences are shown with grey shading.

Additional file 14: Figure S12. laaM/Tms 1-like protein from pTiAB2/73 is more similar to non-T-DNA sequences than to T-DNA-encoded laaM. Alignment of tryptophan 2-monooxygenase -like sequences from pTiAB2/73, from two Mesorhizobium species, from one Rhizobium species ( 90\% identical), one betaproteobacterial oxidoreductase (Dickeya chrysanthemi, 68\% identical), and tryptophan 2-monooxygenases (iaaM/tms 1) from the T-DNAs of the Ti plasmids of A. tumefaciens strains C58, Ach5 (LBA4213) and S4 (46-50\% identical). Residues identical in the majority of sequences are shown with black shading, residues similar in the majority of sequences are shown with grey shading.

Additional file 15: Figure S13. pTiAB2/73 lpt/Tzs-like protein shows strongest similarity to tzs-encoded isopentenyl transferases. Alignment of pTiAB2/73 tzs-like encoded protein to isopentenyl transferases from multiple Ri and Ti plasmids. Sequence identity versus pTiAB2/73 tzs-like varied from 44\% (pTiS4 ipt) to 83\% (pRi2659 tzs). Residues identical in the majority of sequences are shown with black shading, residues similar in the majority of sequences are shown with grey shading.

Additional file 16: Figure S14. Comparison between of a number of Ti plasmid vir regions. Comparison between the vir region of pTiAB2/73 with those of pRi2659 (cucumopine Ri plasmid), pTiCFBP5473 (Ti plasmid from A. larrymoorei strain CFBP5473) and pTiK306 (Ti plasmid from A. vitis strain K306). The tBLASTx hits $(e<0.001)$ are shown in red, with darker bands indicating higher degrees of similarity. Most vir genes are well conserved, but virD3 and virD5, and virE1, virE2 and virE3 only to a lesser extent.

Additional file 17: Table S3. Vir protein similarities. Percentage identities between AB2/73 and other Ti plasmid Vir proteins.

Additional file 18: Table S4.R. etli plasmid p42a contains T-DNA borderlike sequences. This table shows the annotation of a part of $R$. etli plasmid p42a, with in addition to the gene annotation derived from accession NC_007762 the locations of a number of sequences which (almost) match T-DNA border consensus sequences. Also notable is the presence of a NAD/NADP-octopine/nopaline-dehydrogenase-family-protein between two of these borders and the large number of insertion sequences.

Additional file 19: Table S5. Table listing the presence or absence of a vir-box sequence (RYTNCANTTGNAAY) upstream of the virA gene. 


\section{Acknowledgements}

We thank Michael Liem and prof. Herman Spaink for stimulating discussion. Amke den Dulk-Ras, Marcel van Verk and Chris Henkel assisted in the initial stages of the Agrobacterium genome sequencing program of our group.

\section{Authors' contributions}

M.J.G.H. and P.J.J.H. designed the experiments. M.J.G.H. performed the experiments and the bioinformatics/genomics analyses. M.J.G.H. and P.J.J.H. wrote the manuscript text and M.J.G.H. prepared all figures. Both authors reviewed the manuscript. The author(s) read and approved the final manuscript.

\section{Funding}

No external funding.

\section{Availability of data and materials}

The complete genome sequence of AB2/73 was deposited in GenBank under accession numbers CP067071-CP067077. The raw reads are deposited in the Sequence Read Archive (https://www.nlm.nih.gov/sra) under accessions numbers SRR13775335, SRR13775336.

\section{Declarations}

Ethics approval and consent to participate

'Not applicable'

\section{Consent for publication}

'Not applicable'

\section{Competing interests}

The authors declare that they have no competing interests.

Received: 17 June 2021 Accepted: 6 October 2021

Published online: 28 October 2021

\section{References}

1. Bevan MW, Chilton MD. T-DNA of the Agrobacterium Ti and Ri plasmids. Ann Rev Genet. 1982;16:357-84.

2. Kerr A, Panagopoulos CG. Biotypes of Agrobacterium radiobacter var. tumefaciens and their biological control. Phytopath Z. 1977;90:172-9.

3. Lassalle F, Campillo T, Vial L, Baude J, Costechareyre D, Chapulliot D, et al. Genomic species are ecological species as revealed by comparative genomics in Agrobacterium tumefaciens. Genome Biol Evol. 2011;3:762-81.

4. Jumas-Bilak E, Michaux-Charachon S, Bourg G, Ramuz M, Allardet-Servent A. Unconventional genomic organization in the alpha subgroup of the Proteobacteria. J Bacteriol. 1998;180:2749-55.

5. Mousavi SA, Willems A, Nesme X, de Lajudie P, Lindström K. Revised phylogeny of Rhizobiaceae: proposal of the delineation of Pararhizobium gen. nov., and 13 new species combinations. Syst Appl Microbiol. 2015;38:84-90.

6. Kuzmanović N, Smalla K, Gronow S, Puławska J. Rhizobium tumorigenes sp. nov., a novel plant tumorigenic bacterium isolated from cane gall tumors on thornless blackberry. Sci Rep. 2018;8:9051.

7. Otten L. The Agrobacterium phenotypic plasticity (Plast) genes. Curr Top Microbiol Immunol. 2018:418:375-420.

8. Dessaux Y, Petit A, Tempe J. Chemistry and biochemistry of opines, chemical mediators of parasitism. Phytochem. 1993;34:31-8.

9. Lacroix B, Citovsky V. Pathways of DNA transfer to plants from Agrobacterium tumefaciens and related bacterial species. Annu Rev Phytopathol. 2019;25:231-51.

10. Zhu J, Oger PM, Schrammeijer B, Hooykaas PJ, Farrand SK, Winans SC. The bases of crown gall tumorigenesis. J Bacteriol. 2000;182:3885-95.

11. Winans SC. An Agrobacterium two-component regulatory system for the detection of chemicals released from plant wounds. Mol Microbiol. 1991;5:2345-50.

12. Vergunst AC, Schrammeijer B, den Dulk-Ras A, de Vlaam CMT, Regensburg-TuïnkTJG, Hooykaas PJJ. VirB/D4-dependent protein translocation from Agrobacterium into plant cells. Science. 2000;290:979-82.
13. Oger PM, Farrand SK. Co-evolution of the agrocinopine opines and the agrocinopine-mediated control of TraR, the quorum-sensing activator of the Ti plasmid conjugation system. Mol Microbiol. 2001;41:1173-85.

14. Kerr A. Transfer of virulence between isolates of Agrobacterium. Nature. 1969;223:1175-6.

15. Cevallos MA, Cervantes-Rivera R, Gutiérrez-Ríos RM. The repABC plasmid family. Plasmid. 2008;60:19-37.

16. Pinto UM, Pappas KM, Winans SC. The ABCs of plasmid replication and segregation. Nat Rev Microbiol. 2012;10:755-65.

17. Anderson AR, Moore LW. Host specificity in the genus Agrobacterium. Phytopathology. 1978;69:320-3.

18. Smarrelli J, Watters MT, Diba LH. Response of various cucurbits to infection by plasmid-harboring strains of Agrobacterium. Plant Physiol. 1986:82:622-4

19. Unger L, Ziegler SF, Huffman GA, Knauf VC, Peet R, Moore LW, et al. New class of limited-host-range Agrobacterium mega-tumor-inducing plasmids lacking homology to the transferred DNA of a wide-host-range, tumor-inducing plasmid. J Bacteriol. 1985;164:723-30.

20. Otten L, Schmidt JA. T-DNA from the Agrobacterium tumefaciens limitedhost-range strain AB2/73 contains a single oncogene. Mol Plant-Microbe Int. 1998;11:335-42.

21. Lobry JR. Asymmetric substitution patterns in the two DNA strands of bacteria. Mol Biol Evol. 1996;13:660-5.

22. Grigoriev A. Analyzing genomes with cumulative skew diagrams. Nucl Acids Res. 1998:26:2286-90

23. Harrison PW, Lower RPJ, Kim NKD, Young JPW. Introducing the bacterial 'chromid': not a chromosome, not a plasmid. Trends Microbiol. 2010;18:141-8.

24. Slater SC, et al. Genome sequences of three Agrobacterium biovars help elucidate the evolution of multichromosome genomes in bacteria. J Bacteriol. 2009;191:2501-11.

25. Hooykaas MJG, Hooykaas PJJ. The genome sequence of hairy root Rhizobium rhizogenes strain LBA9402: bioinformatics analysis suggests the presence of a new opine system in the agropine Ri plasmid. MicrobiologyOpen. 2021;10:e1180. https://doi.org/10.1002/mbo3.1180.

26. Villaseñor T, Brom S, Dávalos A, Lozano L, Romero D, García-de los Santos A. Housekeeping genes essential for pantothenate biosynthesis are plasmid-encoded in Rhizobium etli and Rhizobium leguminosarum. BMC Microbiol. 2011;11:66.

27. Wetzel ME, Olsen GJ, Chakravartty V, Farrand SK. The repABC plasmids with quorum-regulated transfer systems in members of the Rhizobiales divide into two structurally and separately evolving groups. Genome Biol Evol. 2015;7:3337-57.

28. Canaday J, Gérard JC, Crouzet P, Otten L. Organization and functional analysis of three T-DNAs from the vitopine Ti plasmid pTiS4. Mol Gen Genet. 1992:235:292-303.

29. Chilton WS, Petit A, Chilton MD, Dessaux Y. Structure and characterization of the crown gall opines heliopine, vitopine and ridéopine. Phytochemistry. 2001;58:137-42.

30. Otten L. T-DNA regions from 350 Agrobacterium genomes: maps and phylogeny. Plant Mol Biol. 2021. https://doi.org/10.1007/s111103-021-01140-0.

31. Shao S, van Heusden GPH, Hooykaas PJJ. Complete sequence of succinamopine Ti-plasmid pTiEU6 reveals its evolutionary relatedness with nopaline-type Ti-plasmids. Genome Biol Evol. 2019;11:2480-91.

32. Aragón IM, Pérez-Martínez I, Moreno-Pérez A, Cerezo M, Ramos C. New insights into the role of indole-3-acetic acid in the virulence of Pseudomonas savastanoi pv. savastanoi. FEMS Microbiol Lett. 2014;356:184-92.

33. Turk SC, Nester EW, Hooykaas PJ. The virA promoter is a host-range determinant in Agrobacterium tumefaciens. Mol Microbiol. 1993;7:719-24.

34. Haryono M, Tsai YM, Lin CT, Huang FC, Ye YC, Deng WL, et al. Presence of an Agrobacterium-type tumor-inducing plasmid in Neorhizobium sp. NCHU2750 and the link to phytopathogenicity. Genome Biol Evol. 2018;10:3188-95

35. Hooykaas PJJ, Klapwijk PM, Nuti MP, Schilperoort RA, Rorsch A. Transfer of the Agrobacterium tumefaciens TI plasmid to avirulent Agrobacteria and to Rhizobium ex planta. J Gen Microbiol. 1977;98:477-84.

36. Hooykaas PJJ, van Brussel AAN, van Veen RJM, Wijfelman CA. The expression of Sym plasmids and Ti plasmids in rhizobia and agrobacteria. In: Veeger C, Newton WE, editors. Advances in nitrogen fixation research. Den Haag: Nijhoff/Junk Publ; 1984. p. 661-6. 
37. van Veen RJM, den Dulk RH, Bisseling T, Schilperoort RA, Hooykaas PJJ. Crown gall tumor and root nodule formation by the bacterium Phyllobacterium myrsinacearum after the introduction of an Agrobacterium Ti plasmid or a Rhizobium Sym plasmid. Mol Plant Microbe Int. 1988;1:231-4.

38. van Veen RJM, den Dulk RH, Schilperoort RA, Hooykaas PJJ. Ti plasmid containing Rhizobium meliloti are nontumorigenic on plants, despite proper virulence gene induction and T strand formation. Arch Microbiol. 1989;153:85-9.

39. Broothaerts W, Mitchell H, Weir B, Kaines S, Smith LMA, Yang W, et al. Gene transfer to plants by diverse species of bacteria. Nature. 2005;433:629-33.

40. Wendt T, Doohan F, Mullins E. Production of Phytophthora infestansresistant potato (Solanum tuberosum) utilising Ensifer adhaerens OV14. Transgenic Res. 2012;21:567-78.

41. González V, Santamaría RI, Bustos P, Hernández-González I, Medrano-Soto A, Moreno-Hagelsieb G, et al. The partitioned Rhizobium etli genome: genetic and metabolic redundancy in seven interacting replicons. Proc Natl Acad Sci U S A. 2006;7:3834-9.

42. Lacroix B, Citovsky V. A functional bacterium-to-plant DNA transfer machinery of Rhizobium etli. PLoS Pathog. 2016;12:e1005502.

43. Hooykaas PJJ, den Dulk Ras H, Schilperoort RA. Molecular mechanism of Ti plasmid mobilization by R plasmids. Isolation of Ti plasmids with transposon insertions in Agrobacterium tumefaciens. Plasmid. 1980;4:64-75.

44. Brom S, Girard L, Tun-Garrido C, et al. Transfer of the symbiotic plasmid of Rhizobium etli CFN42 requires cointegration with p42a, which may be mediated by site-specific recombination. J Bacteriol. 2004;186:7538-48.

45. Hoekema A, de Pater BS, Fellinger AJ, Hooykaas PJJ, Schilperoort RA. The limited host range of an Agrobacterium tumefaciens strain extended by a cytokinin gene from a wide host range T-region. EMBO J. 1984;3:3043-7.

46. Buchholz WG, Thomashow MF. Host range encoded by the Agrobacterium tumefaciens tumor-inducing plasmid pTiAg63 can be expanded by modification of its T-DNA oncogene complement. J Bacteriol. 1984;160:327-32.

47. Yanofsky M, Lowe B, Montoya A, Rubin R, Krul W, Gordon M, et al. Molecular and genetic analysis of factors controlling host range in Agrobacterium tumefaciens. Mol Gen Genet. 1985;201:237-46.

48. Hooykaas PJJ, den Dulk RH, Schilperoort RA. The Agrobacterium tumefaciens T DNA gene 6b is an onc gene. Plant Mol Biol. 1988;11:791-4.

49. Helfer A, Pien S, Otten L. Functional diversity and mutational analysis of Agrobacterium 6B oncoproteins. Mol Gen Genomics. 2002;267:577-86.
50. Beringer JE. R factor transfer in Rhizobium leguminosarum. J Gen Microbiol. 1974;84:188-98.

51. Jain C, Rodriguez-R LM, Phillippy AM, Konstantinidis KT, Aluru S. High throughput ANI analysis of 90K prokaryotic genomes reveals clear species boundaries. Nat Commun. 2018;9:5114.

52. Meier-Kolthoff JP, Auch AF, Klenk HP, Göker M. Genome sequence-based species delimitation with confidence intervals and improved distance functions. BMC Bioinformatics. 2013;14:60.

53. Tatusova T, DiCuccio M, Badretdin A, Chetvernin V, Nawrocki EP, Zaslavsky $L$, et al. NCBI prokaryotic genome annotation pipeline. Nucleic Acids Res. 2016;44:6614-24.

54. Huerta-Cepas J, et al. Fast genome-wide functional annotation through orthology assignment by eggNOG-mapper. Mol Biol Evol. 2017;34:2115-22.

55. Xie Z, Tang H. ISEScan: automated identification of insertion sequence elements in prokaryotic genomes. Bioinformatics. 2017;33:3340-7.

56. Darling AE, Mau B, Perna NT. progressiveMauve: multiple genome alignment with gene gain, loss and rearrangement. PLoS One. 2010;5:e11147.

57. Guy L, Kultima JR, Andersson SG. genoPlotR: comparative gene and genome visualization in R. Bioinformatics. 2010;26:2334-5.

58. Alikhan NF, Petty NK, Zakour NLB, Beatson SA. BLAST Ring Image Generator (BRIG): simple prokaryote genome comparisons. BMC Genomics. 2011;12:402.

59. Emms DM, Kelly S. OrthoFinder: solving fundamental biases in whole genome comparisons dramatically improves orthogroup inference accuracy. Genome Biol. 2015;16:157.

60. Katoh S. MAFFT multiple sequence alignment software version 7: improvements in performance and usability. Mol Biol Evol. 2013;30:772-80.

61. Huerta-Cepas J, Serra F, Bork P. ETE 3: reconstruction, analysis, and visualization of phylogenomic data. Mol Biol Evol. 2016;33:1635-8.

\section{Publisher's Note}

Springer Nature remains neutral with regard to jurisdictional claims in published maps and institutional affiliations.
Ready to submit your research? Choose BMC and benefit from:

- fast, convenient online submission

- thorough peer review by experienced researchers in your field

- rapid publication on acceptance

- support for research data, including large and complex data types

- gold Open Access which fosters wider collaboration and increased citations

- maximum visibility for your research: over 100M website views per year

At BMC, research is always in progress.

Learn more biomedcentral.com/submissions 